\section{EPISTASIS EN VARIEDADES SINTÉTICAS DE MAÍZ}

\section{EPISTASIS IN MAIZE SYNTHETIC VARIETIES}

\section{Fidel Márquez Sánchez}

Centro Regional Universitario de Occidente, Universidad Autónoma Chapingo. Rosario Castellanos, Col. Residencial La Cruz. 44950, Guadalajara, Jalisco. Fax: 013336467794.

* Autor para correspondencia (fidelmqz@hotmail.com)

\section{RESUMEN}

En un artículo anterior se presentaron los cálculos de la epistasis para varias poblaciones de plantas de maíz (Zea mays $L$.) frecuentes en el mejoramiento genético, y se mostró cómo actúa este fenómeno genético de acuerdo con la estructura de las poblaciones. Dado que los maíces sintéticos poseen amplio potencial para ser aprovechados por los campesinos marginados, y dada la relativa facilidad que tienen en su obtención, en este artículo se calcula la epistasis promedio de variedades sintéticas constituidas por líneas homocigóticas bajo el modelo epistático.

Palabras clave: Zea mays, epistasis, variedad sintética.

\section{SUMMARY}

In a previous article the equations for estimating epistasis were developed for several populations frequently used in maize (Zea mays L.) breeding, and it was shown how this genetic phenomena acts according to the structure of the involved populations. Since synthetic maize varieties have a big potential to be utilized by marginalized farmers and are relatively easy to obtain, this article focuses on calculating the mean epistasis of a synthetic variety created from homozygous lines under the epistatic model.

Index words: Zea mays, epistasis, synthetic variety.

\section{INTRODUCCIÓN}

Las variedades sintéticas o sintéticos de maíz (Zea mays L.) se han propuesto como una alternativa al uso de híbridos. La principal razón es el alto costo de la semilla de los híbridos, que en las áreas maiceras por excelencia es hasta de más de dos mil pesos por hectárea, (precios de 2010 , en pesos mexicanos) y representa hasta $15 \%$ de los costos de producción. En estas variedades sintéticas (también denominados "sintéticos") es necesario conocer cómo las afecta la epistasis, ya que en un artículo previo Márquez-Sánchez (2009) demostró que la epistasis explica, por ejemplo, la superioridad de un híbrido de cruza simple sobre el híbrido carente de ella.

Márquez-Sánchez (1992) y Sahagún-Castellanos (1994) demostraron que los sintéticos pueden obtenerse desde la generación inicial o generación cero, mediante un compuesto elaborado con las líneas que se desean sintetizar, y que luego se somete a polinización aleatoria. Márquez-Sánchez (2008) y Sahagún-Castellanos (2003) también demostraron que los sintéticos pueden hacerse con otros materiales genéticos, como híbridos simples e híbridos dobles, aunque los sintéticos resultantes tienen diferentes coeficientes de endogamia.

\section{MATERIALES Y MÉTODOS}

Se seguirá el método que propusieron Márquez-Sánchez (1992) y Sahagún-Castellanos (1994), en el que las líneas se sintetizan a partir de la generación cero mediante polinización aleatoria, para los sintéticos de 2, 3, 6 y n líneas, para después seguir la metodología de MárquezSánchez (2009) en lo que se refiere a los coeficientes de los efectos epistáticos.

\section{RESULTADOS \\ Sintético de dos líneas}

Sean las dos líneas homocigóticas $\mathrm{AxAxB} \mathrm{B}_{\mathrm{B}}$ y $A_{w} A_{w} B_{\kappa} B_{\kappa}$, que dan lugar al híbrido simple (HS), con las que se hace el compuesto y cuyos gametos son $A_{x B}$ y $\mathrm{AwB}$.

La unión aleatoria de gametos producirá la progenie que se muestra en el Cuadro 1.

\begin{tabular}{lcc}
\multicolumn{3}{c}{ Cuadro 1. Progenie de un sintético con dos líneas. } \\
\hline $\mathrm{AxB}_{\mathrm{J}}$ & $\mathrm{Aw}_{\mathrm{w}} \mathrm{B}_{\mathrm{J}}$ \\
$\mathrm{AwB}_{\mathrm{w}}$ & $\mathrm{AxA}_{\mathrm{x}} \mathrm{B}_{\mathrm{J}} \mathrm{B}_{\mathrm{J}}$ & $\mathrm{AxA}_{w} \mathrm{~B}_{\mathrm{J}} \mathrm{B}_{\mathrm{K}}$ \\
\hline
\end{tabular}

Se puede observar en el Cuadro 1 que en la progenie sólo hay dos genotipos homocigotes dobles más un heterocigote por duplicado, y como para cada genotipo hay seis efectos genéticos, el número total de efectos es 4 x $6=24$. Dichos efectos son:

$$
\begin{aligned}
& A x A x B B_{J}=2 \alpha^{A} x+\delta^{A} x x+2 \alpha^{B}+\delta^{B}{ }_{J J} \\
& A w A w B_{k} B_{K}=2 \alpha^{A}{ }^{A}+\delta^{A} w w+2 \alpha^{B_{K}}+\delta^{B}{ }_{K K} \\
& 2\left(\mathrm{Ax} A w \mathrm{BJ}_{\mathrm{BK}}\right)=2 \alpha^{\mathrm{A}} \mathrm{x}+2 \alpha^{\mathrm{A}} \mathrm{w}+2 \delta^{\mathrm{A}} \mathrm{xw}+2 \alpha^{\mathrm{B}}+2 \alpha^{\mathrm{B}}{ }_{\mathrm{K}} \\
& +2 \delta^{\mathrm{B}} \mathrm{JK}
\end{aligned}
$$


Cuadro 3. Producción de un sintético con cuatro líneas.

\begin{tabular}{|c|c|c|c|c|}
\hline & $\mathrm{AxBJ}$ & $\mathrm{AwBK}$ & ANBo & $\mathrm{AyBp}$ \\
\hline $\mathrm{AxB}$ & $\mathrm{AxAxB}_{\mathrm{A}} \mathrm{B}_{\mathrm{J}}$ & $\mathrm{AxAwB \jmath Bк}$ & AxAnBsBo & $\mathrm{AxAyBJBp}$ \\
\hline AwBK & $\mathrm{AxAwB} \mathrm{B}_{\mathrm{J}}$ & АwАwВкВк & $\mathrm{Aw} \mathrm{AnB}_{\mathrm{N}} \mathrm{B} о$ & АwАүВкВр \\
\hline $\mathrm{ANBo}_{0}$ & $\mathrm{AxAn}_{\mathrm{N}} \mathrm{B}_{\mathrm{B}}$ & $\mathrm{AN}_{\mathrm{N}} \mathrm{AwBoBk}$ & $\mathrm{An}_{\mathrm{N}} \mathrm{AnBoBo}$ & $\mathrm{AnN}_{\mathrm{N}} \mathrm{B}_{\mathrm{B}} \mathrm{B}$ \\
\hline AyBp & $\mathrm{Ay}_{\mathrm{AxB}} \mathrm{B}_{\mathrm{BP}}$ & $\mathrm{Ar}_{\mathrm{AwB}} \mathrm{BK}_{\mathrm{K}}$ & $\mathrm{Ay}_{\mathrm{AnBoB}} \mathrm{Bp}$ & $\mathrm{Ay}_{\mathrm{Ay}} \mathrm{BoB}$ \\
\hline
\end{tabular}

Al obtener todos los efectos estadísticos genéticos y su acumulación, los coeficientes unificados quedarán:

$\mathrm{Ms}_{4 \mathrm{~L}}=\mathrm{m}+\alpha^{\mathrm{A}} \mathrm{X}+\alpha^{\mathrm{A}}{ }_{\mathrm{M}}+\alpha^{\mathrm{A}}{ }_{\mathrm{N}}+\alpha^{\mathrm{A}} \mathrm{Y}+\alpha^{\mathrm{B}}{ }_{\mathrm{J}}+\alpha^{\mathrm{B}}{ }_{\mathrm{K}}+$ $\alpha^{\mathrm{B}}+\alpha^{\mathrm{B}}+1 / 8 \delta^{\mathrm{A}} \mathrm{xx}+1 / 8 \delta^{\mathrm{A}} \mathrm{ww}^{\mathrm{A}}+1 / 8 \delta^{\mathrm{A}} \mathrm{NN}^{\mathrm{A}}+1 / 8$ $\delta^{\mathrm{A}} \mathrm{YY}+1 / 8 \delta^{\mathrm{B}} \mathrm{JJ}+1 / 8 \delta^{\mathrm{B}} \mathrm{KK}^{\mathrm{A}}+1 / 8 \delta^{\mathrm{B}}{ }_{\mathrm{OO}}+1 / 8 \delta^{\mathrm{B}}{ }_{\mathrm{PP}}+1 / 4$ $\delta^{\mathrm{A}} \mathrm{XW}+1 / 4 \delta^{\mathrm{A}} \mathrm{XN}+1 / 4 \delta^{\mathrm{A}} \mathrm{XY}+1 / 4 \delta^{\mathrm{A}} \mathrm{wN}^{\mathrm{A}}+1 / 4 \delta^{\mathrm{A}} \mathrm{wY}^{\mathrm{A}}+$ $1 / 4 \delta^{\mathrm{A}}{ }_{\mathrm{NY}}+1 / 4 \delta^{\mathrm{B}} \mathrm{JK}+1 / 4 \delta^{\mathrm{B}} \mathrm{JO}+1 / 4 \delta^{\mathrm{B}}{ }_{\mathrm{JP}}+1 / 4 \delta^{\mathrm{B}} \mathrm{KO}+$ $1 / 4 \delta^{\mathrm{B}}{ }_{\mathrm{KP}}+1 / 4 \delta^{\mathrm{B}}{ }_{\mathrm{OP}}+16 \alpha \alpha+2 \alpha \delta$ (DomHom) $+6 \alpha \delta$ (DomHet) $+2 \delta \alpha$ (DomHom) $+6 \delta \alpha$ (DomHet) $+1 / 4$ $\delta \delta($ DomHom x DomDom $)+3 / 4 \delta \delta($ DomHom x DomHet $)+$ $3 / 4 \delta \delta($ DomHet x DomHom) $+9 / 4 \delta \delta($ DomHet x DomHet)

\section{Sintético de n líneas}

Con referencia primero a los efectos estadísticos genéticos del locus A, éste será similar a los efectos del locus B. En S2L y en S3L se puede ver que los efectos $\alpha$ son 2 y 3 respectivamente, y los efectos $\delta$ son 1 y $3 / 2$, respectivamente, lo que sirve para cotejar que el número de efectos dominantes es la mitad de los efectos aditivos. Como esencialmente el cuadro de apareamiento aleatorio para cualquier número de líneas no cambia, entonces para los sintéticos de dos y tres líneas se tiene la información del Cuadro 4.

Cuadro 4. Efectos $\alpha$ y $\delta$ conforme el número de líneas de los sintéticos y sus respectivos coeficientes.

\begin{tabular}{lcccccc}
\hline Efectos & \multicolumn{2}{c}{ Números de líneas } & \multicolumn{3}{c}{ Coeficientes } \\
\hline$\alpha$ & 2 & 3 & 4 & 1 & 1 & 1 \\
$\delta$ & 1 & $3 / 2$ & 2 & $1 / 4,1 / 2$ & $1 / 6,1 / 3$ & $1 / 8,1 / 4$ \\
\hline
\end{tabular}

Esta información muestra que los valores de los efectos $\alpha$ son el doble de los efectos $\delta$, de manera que para números mayores de líneas dichos valores serán: 5 y 2.5 , 6 y 3,7 y 3.5 , etc. Además, se puede ver que al ignorar a sus coeficientes, el número de veces en que aparece un efecto del tipo $\delta x x$ es el mismo del número de líneas, y que el denominador de su coeficiente es el doble de dicho número, o sea: $\mathrm{n}(1 / 2 \mathrm{n})$, lo cual siempre es igual a $1 / 2$, cómo se muestra en el Cuadro 5. El número de veces del tipo $\delta x x$, sin considerar a sus coeficientes, es el número de combinaciones de $\mathrm{n}$ tomadas de 2 en 2 y multiplicado por $(1 / 2 n)$, es decir: $[n(n-1) / 2](1 / 2 n)=(n-1) / 2$.

Como los efectos $\delta$ incluyen tanto a los DomHom como a los DomHet, se pueden inferir los coeficientes para números mayores de líneas, como se muestra en el Cuadro 5.

Cuadro 5. Número de tipos dominantes y sus coeficientes, según el número de líneas usadas en los sintéticos.

\begin{tabular}{|c|c|c|c|c|}
\hline \multirow{2}{*}{$\begin{array}{l}\text { Número } \\
\text { de líneas }\end{array}$} & \multicolumn{2}{|c|}{ Números } & \multicolumn{2}{|c|}{ Coeficientes } \\
\hline & Tipo $\delta x x$ & Tipo $\delta$ xw & Tipo $\delta x x$ & Tipo $\delta \mathrm{xw}$ \\
\hline 2 & $1 / 2$ & $1 / 2$ & $1 / 4$ & $1 / 2$ \\
\hline 3 & $1 / 2$ & $2 / 2$ & $1 / 6$ & $1 / 3$ \\
\hline 4 & $1 / 2$ & $3 / 2$ & $1 / 8$ & $1 / 4$ \\
\hline 5 & $1 / 2$ & $4 / 2$ & $1 / 10$ & $1 / 5$ \\
\hline 6 & $1 / 2$ & $5 / 2$ & $1 / 12$ & $1 / 6$ \\
\hline
\end{tabular}

Con esta información ya se conocen los números de líneas Tipo $\delta \mathrm{xx}$ y Tipo $\delta \mathrm{xw}$, con los cuales se puede comprobar que sus sumas (Tipo $\delta \mathrm{xx}+$ Tipo $\delta \mathrm{xw}$ ) son la mitad de los números de los efectos $\alpha$; así, para 2, 3, 4, 5 y 6 líneas, se tendrá:

$1 / 2+1 / 2=1,1 / 2+1=3 / 2,1 / 2+3 / 2=2,1 / 2+2$ $=5 / 2$, y $1 / 2+5 / 2=3$.

De acuerdo con los coeficientes, al aumentar el número de líneas los efectos Tipo $\delta x x$ tienden a desaparecer, así como los efectos Tipo $\delta$ xw que desaparecen a una tasa del doble de los efectos Tipo $\delta x x$. Es decir, al aumentar el número de líneas que conforman un sintético los efectos DomHom tienden a desaparecer, pero siempre tienen un número igual a $1 / 2$, y los efectos DomHet resultan con números superiores a $1 / 2$, iguales a $(n-1) / 2$. Esto sucedería en una variedad de polinización libre, en la cual habría siempre $1 / 2$ de los efectos Tipo $\delta x x$ y casi el doble de efectos del Tipo $\delta$ xw.

Por tanto, con los valores de los coeficientes de los efectos genéticos dados en el Cuadro 4, y con los coeficientes de los efectos $\delta$ de los Tipos $\delta$ xx y Tipo $\delta$ xw, dados en el Cuadro 5, se está en posición de calcular los efectos estadísticos $\alpha$ y $\delta$; sin embargo, queda pendiente calcular las interacciones epistáticas.

En los sintéticos de 2, 3 y 4 líneas se puede ver que los coeficientes de efectos epistáticos obedecen a un binomio al cuadrado cuyos términos son los coeficientes de $\alpha$ y $\delta$; dichos coeficientes se simbolizan como tales en lo que sigue, de manera que para los efectos epistáticos se 
tendrá: $(\alpha+\delta)^{2}=\alpha \alpha+\alpha \delta+\delta \alpha+\delta \delta$. El número de efectos $\alpha$ fue fijado por el investigador, y se sabe que la suma de los números de los efectos $\delta$ es la mitad del de los efectos $\alpha$, de manera que con esta información se pueden calcular sus respectivos coeficientes y así poder realizar el binomio. Se tendrá entonces:

$\mathrm{M}_{\mathrm{SnL}}=\mathrm{m}+\alpha^{\mathrm{A}} \mathrm{x}+\alpha^{\mathrm{A}} \mathrm{W}+\ldots+\alpha^{\mathrm{A}}{ }_{\mathrm{n}}+\alpha^{\mathrm{B}}{ }_{\mathrm{J}}+\alpha^{\mathrm{B}_{\mathrm{K}}}+\ldots$ $+\alpha^{\mathrm{B}} \mathrm{n}+1 / 2 \mathrm{n} \delta^{\mathrm{A}} \mathrm{xx}+1 / 2 \mathrm{n} \delta^{\mathrm{A}}{ }_{\mathrm{wW}}+\ldots+1 / 2 \mathrm{n} \delta^{\mathrm{A}}{ }_{\mathrm{nn}}+$ $1 / 2 \mathrm{n} \delta^{\mathrm{B}}{ }_{\mathrm{JJ}}+1 / 2 \mathrm{n} \delta^{\mathrm{B}}{ }_{\mathrm{KK}}+\ldots+1 / 2 \mathrm{n} \delta^{\mathrm{B}}{ }_{\mathrm{nn}}+1 / \mathrm{n} \delta^{\mathrm{A}} \mathrm{XW}+\ldots$ $+1 / \mathrm{n}^{\mathrm{A}}(\mathrm{n}-1) \mathrm{n}+1 / \mathrm{n} \delta^{\mathrm{B}}{ }_{\mathrm{JK}}+\ldots+1 / \mathrm{n} \delta_{(\mathrm{n}-1) \mathrm{n}}^{\mathrm{B}}$

$+n^{2} \alpha \alpha+\left(n^{2} / 2\right) \alpha \delta+\left(n^{2} / 2\right) \delta \alpha+(n / 2)^{2} \delta \delta$

Por ejemplo, para el caso de $\mathrm{n}=3$, se tendrá: $(3+$ $3 / 2)^{2}=9 \alpha \alpha+4.5 \alpha \delta+4.5 \delta \alpha+2.25 \delta \delta$, lo cual puede comprobarse al examinar S3L.

\section{DISCUSIÓN}

¿Qué sucede si se sigue incrementando el número de líneas por arriba de 4? Córdova y Márquez (1979) hicieron los sintéticos posibles con ocho líneas, y fueron comparados en cinco localidades. El número de líneas varió de uno en uno, es decir, $\mathrm{n}=2,3, \ldots, 8$. Se establecieron varios grupos dentro de cada $\mathrm{n}$; por ejemplo, cuando $\mathrm{n}=3$, dentro de este grupo hubo ( 3 $\mathrm{x} 2) / 2=3$ sintéticos (obviamente para $\mathrm{n}=8$, sólo hubo un sintético). El agrupamiento se hizo con el propósito de no escoger al azar sólo un sintético para cada n, el cual representaría al promedio del grupo de sintéticos en cuestión, sino que para lograr mayor precisión estadística, el método usado de hecho representaría al sintético de cada $\mathrm{n}$.

Con este procedimiento se graficaron los rendimientos de los sintéticos sobre sus $\mathrm{n}$ y se encontró que conforme ésta aumentaba también se incrementaban sus rendimientos, y se espera que la gráfica sea asintótica. Este resultado se debe al incremento de los efectos $\alpha^{\mathrm{A}} \mathrm{x}$ y Tipo $\delta^{\mathrm{A}} \mathrm{XY}$, pero no al de los efectos Tipo $\delta^{\mathrm{A}} \mathrm{Xx}$, los que, como puede apreciarse adelante, reducen el rendimiento de la segunda generación con respecto al de la primera generación en los híbridos simples, los que dan lugar a los sintéticos por el apareamiento aleatorio de su compuesto.

Con respecto a la segunda generación $\left(\mathrm{F}_{2}\right)$ de un híbrido simple (HS) proveniente de su apareamiento aleatorio, en base en lo descrito en Márquez-Sánchez (2009), la media del HS ( $\left.\mathrm{AxB} \mathrm{B}_{\mathrm{A}} \mathrm{ABk}\right)$ es:

$$
\begin{gathered}
(\mathrm{HS})=\mathrm{m}+\alpha^{\mathrm{A}} \mathrm{x}+\alpha^{\mathrm{A}} \mathrm{w}+\delta^{\mathrm{A}} \mathrm{xW}+\alpha^{\mathrm{B}}+\alpha^{\mathrm{B}}{ }_{\mathrm{K}}+\delta^{\mathrm{B}}{ }_{\mathrm{JK}} \\
+\alpha \alpha \mathrm{XJ}+\alpha \alpha_{\mathrm{XK}}+\alpha \alpha_{\mathrm{WJ}}+\alpha \alpha_{\mathrm{WK}}
\end{gathered}
$$

$$
\begin{aligned}
& +\alpha \delta \text { хJк }+\alpha \delta \text { wJK }+\delta \alpha \text { xwJ }+\delta \alpha \text { xwK } \\
& +\delta \delta \text { хwנк }
\end{aligned}
$$

La segunda generación del HS (o $\mathrm{F}_{2}$ ) se obtiene de su

\begin{tabular}{|c|c|c|c|c|}
\hline & $\mathrm{AxBJ}$ & $\mathrm{AxBK}$ & AwBJ & $\mathrm{AwBK}_{\mathrm{K}}$ \\
\hline $\mathrm{AxBJ}$ & AxAxBзBл & $\mathrm{AxAxB \jmath Bк}$ & $\mathrm{AxAwB \jmath B}$ & $\mathrm{AxAwB \jmath Bк}$ \\
\hline $\mathrm{AxBk}$ & $\mathrm{Ax} A x \mathrm{B \jmath}_{\mathrm{J}}$ & $\mathrm{AxAxBкBк}$ & $\mathrm{AxAwB \jmath Bк}$ & AxAwBкBк \\
\hline $\mathrm{AwB}$ & AxAwBıBл & $\mathrm{AxAwB \jmath Bк}$ & $\mathrm{Aw} \mathrm{Aw}_{\mathrm{w}} \mathrm{B}_{\mathrm{J}}$ & AwAwBıBк \\
\hline AwBk & AxAwBगBк & AxAwBкBк & AwAwBıВк & AwAwBкBk \\
\hline
\end{tabular}
apareamiento aleatorio mostrado en el Cuadro 6.

Cuadro 6. Progenie de la $F_{2}$ de un híbrido simple obtenida por apareamiento aleatorio.

Cada uno de los 16 genotipos del Cuadro 6, tiene seis efectos estadísticos genéticos, por lo que el total de éstos es $16 \times 6=96$, cuyas respectivas sumas de efectos son: $\alpha^{\mathrm{A}} \mathrm{X}=\alpha^{\mathrm{A}} \mathrm{W}=\alpha^{\mathrm{B}}=\alpha^{\mathrm{B}}{ }_{\mathrm{K}}=16 ; \delta^{\mathrm{A}} \mathrm{xx}=\delta^{\mathrm{A}} \mathrm{wW}=\delta^{\mathrm{B}} \mathrm{JJ}=$ $\delta^{\mathrm{B}} \mathrm{KK}=4 ; \mathrm{y} \delta^{\mathrm{A}} \mathrm{xW}=\delta^{\mathrm{B}} \mathrm{JK}=8$. Con los efectos se puede obtener la media genotípica una vez que se han unificado, y la media queda como sigue:

$\mathrm{F}_{2}(\mathrm{HS})=\mathrm{m}+\alpha^{\mathrm{A}} \mathrm{x}+\alpha^{\mathrm{A}} \mathrm{w}+1 / 4 \delta^{\mathrm{A}} \mathrm{xx}+1 / 4 \delta^{\mathrm{A}} \mathrm{ww}^{\mathrm{A}}+1 / 2$

$\delta^{\mathrm{A}} \mathrm{XW}+\alpha^{\mathrm{B}}+\alpha^{\mathrm{B}}{ }_{\mathrm{K}}+1 / 4 \delta^{\mathrm{A}} \mathrm{JJ}+1 / 4 \delta^{\mathrm{B}}{ }_{\mathrm{KK}}+1 / 2 \delta^{\mathrm{B}} \mathrm{JK}+\mathrm{I}$

en donde I representa a las interacciones epistáticas.

Como se puede apreciar, la media de la $\mathrm{F}_{2}(\mathrm{HS})$ es la misma que la del S2L, de manera que se puede concluir que el S2L tiene la misma reducción en rendimiento que la $\mathrm{F}_{2}(\mathrm{HS})$. Por tanto, si este resultado se hace extensivo a sintéticos derivados de cualquier número (n) de líneas, sus rendimientos siempre serían menores que los de los híbridos usados como progenitores.

Por otra parte, como los sintéticos fueron obtenidos con base en el apareamiento aleatorio del compuesto de las líneas involucradas, habría un incremento del rendimiento, del de las líneas al del sintético; esto se explica con base en el rendimiento de los híbridos que surgen dentro del sintético, pero este incremento es igual a la reducción del sintético aquí concebido a partir de los híbridos.

Si ahora se examina la media del S2L dada atrás, se puede ver que todos los efectos estadísticos genéticos del primero están contenidos en el segundo; pero en el S2L existen además los efectos Tipo $\delta x x$ y las interacciones $\alpha \delta \mathrm{xx}$ y $\delta$ Js $\alpha$; las interacciones $\delta \delta$ siempre son de los Tipos

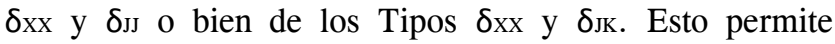
demostrar que la reducción del rendimiento en S2L, al ser comparado con HS, se debe a la presencia de los efectos $\delta$ homocigóticos, lo cual puede hacerse extensivo a sintéticos con números mayores de líneas. 


\section{CONCLUSIONES}

Dado el papel que las interacciones epistáticas juegan en el rendimiento de las variedades sintéticas de maíz, de antemano no puede saberse su manera de actuar en dicho sentido. Sólo cuando se asignen valores particulares a las frecuencias génicas de las líneas involucradas se podrá saber cómo actuarán las interacciones epistáticas. Con los resultados del presente artículo se concluye que conforme aumenta el número de líneas se incrementará el rendimiento medio de los sintéticos, debido al incremento de los efectos aditivos, de las interacciones aditivo $\mathrm{x}$ aditivo y de los efectos dominantes heterocigóticos, aunque también habrá una reducción, de menor grado, por la presencia de los efectos dominantes homocigóticos.

\section{BIBLIOGRAFÍA}

Córdova-Orellana H S, F Márquez S (1979) Efecto del número de líneas endogámicas sobre el comportamiento de variedades sintéticas derivadas de una población de maíz (Zea mays L.). I: Rendimiento. Agrociencia 37:235-252.

Márquez-Sánchez F (1992) Inbreeding and yield prediction in synthetic maize cultivars made with parental lines. I. Basic methods. Crop Sci. 32:345-349.

Márquez-Sánchez F (1993) Inbreeding and yield prediction in synthetic cultivars of maize. II. Alternative methods. Crop Sci. 33:1-5.

Márquez-Sánchez F (2008) Endogamia y predicción de sintéticos de maíz de cruzas dobles. Rev. Fitotec. Mex. 31 (Número esp. 3):1-4.

Márquez-Sánchez F (2009) La epistasis en los híbridos y en la retrocruza en maíz. In: Memoria de la III Reunión Nacional para el Mejoramiento, Conservación y Uso de los Maíces Criollos. Instituto Tecnológico de Roque y Sociedad Mexicana de Fitogenética. 14-16 de octubre de 2009. Celaya, Gto. pp:215 .

Sahagún-Castellanos J (1994) Sobre el cálculo de la endogamia de variedades sintéticas. Agrociencia S. Fitociencia 5:67-78.

Sahagún-Castellanos J (2003) Coeficiente de endogamia de las variedades sintéticas de cruzas dobles. Agrociencia 37:641-655. 